\title{
Managing Irrigation in Indian Agriculture Using Fuzzy Logic - A Decision Support System
}

\author{
N. Suruthi *, R. Saranya, S. Subashini, P. Shanthi, A. Umamakeswari \\ Department of CSE, SASTRA Deemed to be University, Thanjavur \\ *Corresponding Author Email: suruthi.sastra28@gmail.com
}

\begin{abstract}
Supplementation of water for irrigation in needed in south India due to uncertainty of monsoon rainfall. This paper proposes a support system to manage the irrigation system based on the information provided by humidity, temperature, soil moisture and weather information. The temperature, humidity and soil moisture data were collected by sensors. The proposed ANFIS based system consists of $\mathrm{N}$ inputs and a single output which determines the irrigation time needed for the crop. The experimentation is carried out using real time data collected from the region of VALLAM, located near THANJAVUR. The result helps in determining the time for irrigation which helps in increasing the yield of the crop.
\end{abstract}

Keywords: Irrigation Decision Support System, Sensors, Fuzzy Logic, Adaptive Neuro Fuzzy Inference Systems.

\section{Introduction}

Agriculture is important in Indian economy. It is a common and well known fact that agriculture is the backbone of TAMILNADU. People are very much dependent on the agriculture. We can also see various developments in the agriculture techniques of TAMILNADU people. With the advancement in technology, people are focusing on the various new techniques and new stuff to get good yield for their crops. Once upon a time, there were people in our state who were fully dedicated only to agricultural development. People are focusing more on the betterment of their life and individuality and this idea makes them forget about the agriculture. When Crops are grown in fields, it is very much necessary that the fields should water properly based on the needs. There are also some crops that are present which ultimately needs excess amount of water for the growth and the enrichment.

Irrigation is the major factor for agriculture in TAMILNADU. In today's world water is the most demanding source of energy. Recently there was a big loss in agriculture because of the lack of rain and water. In most cases the agriculture lands were kept without proper irrigation and proper maintenances for many years and this led to a condition in which the land was made unfit for the agriculture. Even though water was one of the reasons for the same, the people were the other main reason for the agriculture to get affected. This made no source of water for the land and this is the main reason why the irrigation system in our state was affected by great extent. The proposed work gives a decision support system which uses sensors to collect Humidity, Temperature and soil moisture data. An advice is made based on the time needed to irrigate the crop. Expert agronomist is consulted for analyzing the information from different sources. Finally, an irrigation report is provided based on the information collected, which indicates the time needed to irrigate the crop.

\section{Related work}

This section describes a general analysis and the works carried in water prediction. Agricultural system of Algeria [1] has used Internet of Things along with smart technologies to minimize the water consumption and to create a remote based monitoring of the irrigation process. But it increases complexity because of the use of network connections, and focused more on establishing the WSN. It could have been simpler. Traditional methodology along with latest technologies as Internet of Things and Wireless sensor networks [2] used for monitoring agriculture. System used PIR sensors to monitor heat, but this does not go well along with raspberry pi. So Arduino can be used as it provides more compatibility along with the sensor, and it is also very cheaper.

Arduino based irrigation system proposed in [3] uses a soil sensor which gives a voltage signal concerning with the humidify level in the field soil and tested against the threshold value which is taken by various soils and specific crops. The drawback in this system is that, they have used soil moisture sensor as the only factor for automated irrigation system. In [4], a advanced novel routing protocol for WSN named ECHERP is used and the system is expected to efficiently manage water supply in agricultural land in an automated way. Even though it manages irrigation, the effect of field characteristics on the quantity of water is not considered. Water prediction is done by concentrating more on watering and roofing system based on the statistical data collected from the sensors, since they may not be accurate due to noises, Kalman filtering [5] is used to smooth the sensor data. A decision tree model is used to take decision based on the sensed value and also the weather information. Decision is made without considering the type of soil, and this is important since different types of soil require different type of irrigation system. 
Fuzzy Decision Support System [6] based on the information provided by crop and site characteristics to improve the irrigation process. They used an existing agricultural model and database. The drawback in this system is absence of dedicated sensors or actuators. Work [7], focused on the Wireless Sensor Networks applications and the challenges and issues associated with it for improving the irrigation system. Scalability, information security is improved and fault tolerance is provided. But, the cost and maintenance for wireless sensor networks is increased. An irrigation decision support system [8] is executed to manage the irrigation system. Machine learning techniques by gathering information of soil and climatic variables is used. Weather forecast information is not added and it is not suitable for several conditions and regions.

\section{Sensor description}

The first part of the proposed system architecture is to collect the basic information needs like temperature, humidity and soil moisture data. The temperature and humidity data were collected using DHT11 sensor (Fig.1 and Fig.2) since it is both cost effective and provides fast response for monitoring both temperature and humidity data. The DHT11 sensor data is connected to ARDUINO using jumper wires and the ARDUINO is connected to the laptop using the cable for processing. The reading of temperature and humidity data is displayed in the ARDUINO IDE. This information is noted for further processing. Similarly, the soil moisture data for the particular agricultural field is collected using the soil sensor (kits Guru soil moisture sensor module), and it is placed in the agricultural area for taking the humidity content of the soil.

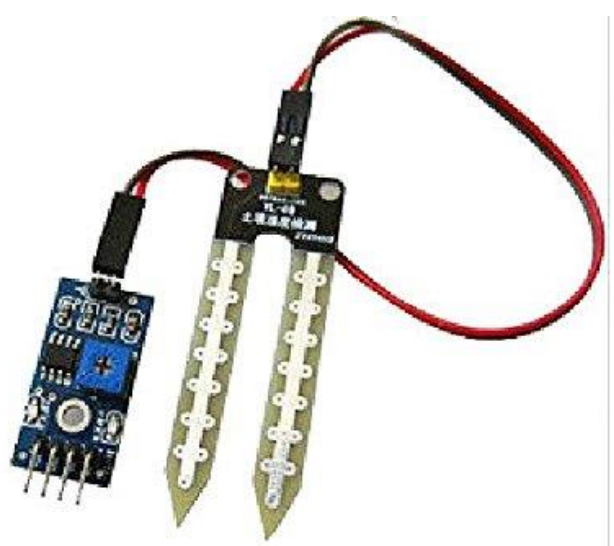

Fig.1: Soil sensor

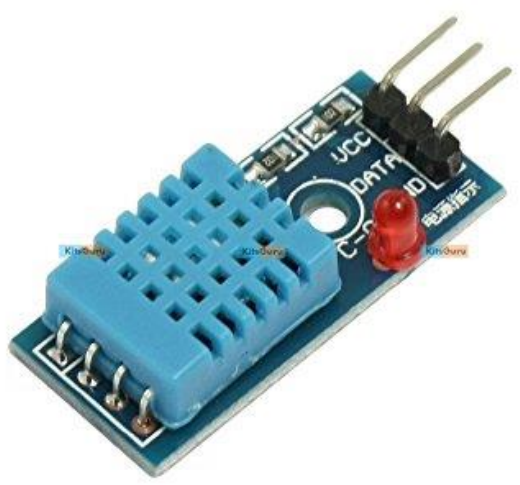

Fig. 2: DHT11 sensor

\section{Experimental setup}

The experiment is carried out in the region of VALLAM, located near THANJAVUR. Paddy crops are grown in this field and the size of the field is $1 / 4$ acre. The Fig. 3 shows the agricultural field in VALLAM. The time required to irrigate the paddy crops is about 90 days. The first 25 to 30 days is used for preparation and transplantation of field and the last 15 days is left dry without any water for harvesting. The average height of the crop in the last 15 days will be $30 \mathrm{~cm}$.

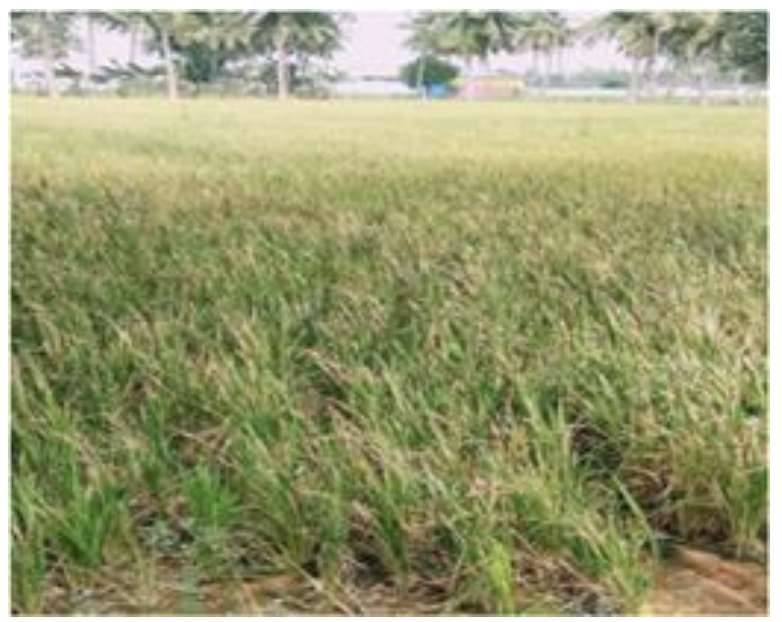

Fig. 3: Agricultural Field

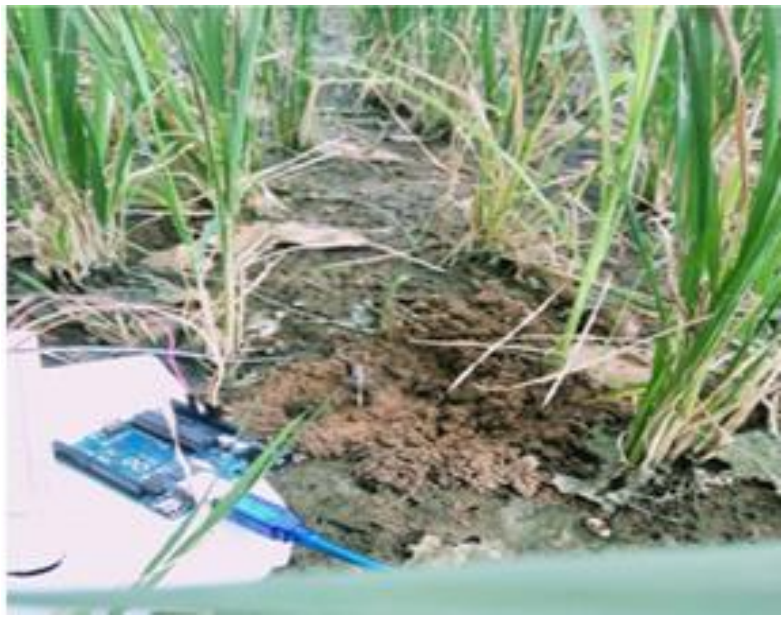

Fig. 4: Measuring Soil Sensor values

The soil sensor is placed at 3 to 4 places in the agricultural area for getting the humidity content of the soil. The soil sensor is kept at three different depths like 1,3 and $5 \mathrm{~cm}$. The readings for various depths are noted. The soil moisture range in dry soil is between 900 and 1000 approximately and when the soil becomes wet the range falls down to 200 . The soil moisture range is noted down for this particular field and it is used for further processing. Fig. 4 shows the soil sensor placed in the agricultural field.

\section{Irrigation prediction}

Irrigation time is predicted using the technique Adaptive Neuro Fuzzy Inference System (ANFIS). It is the combination of fuzzy logic and artificial neural networks. Generally fuzzy logic is an addition of the multivalued logic. The other part is artificial neural network which is in same way with the biological neural networks.

The fuzzy logic implementation is done by MATLAB, using a fuzzy logic designer tool available in the software. The Fig.5 
shows the number of input and output parameters. The input parameters include Temperature, Humidity, Soil Moisture,
Weather forecast and the Various Stages of crops. It provides a single output parameter called Irrigation Time.

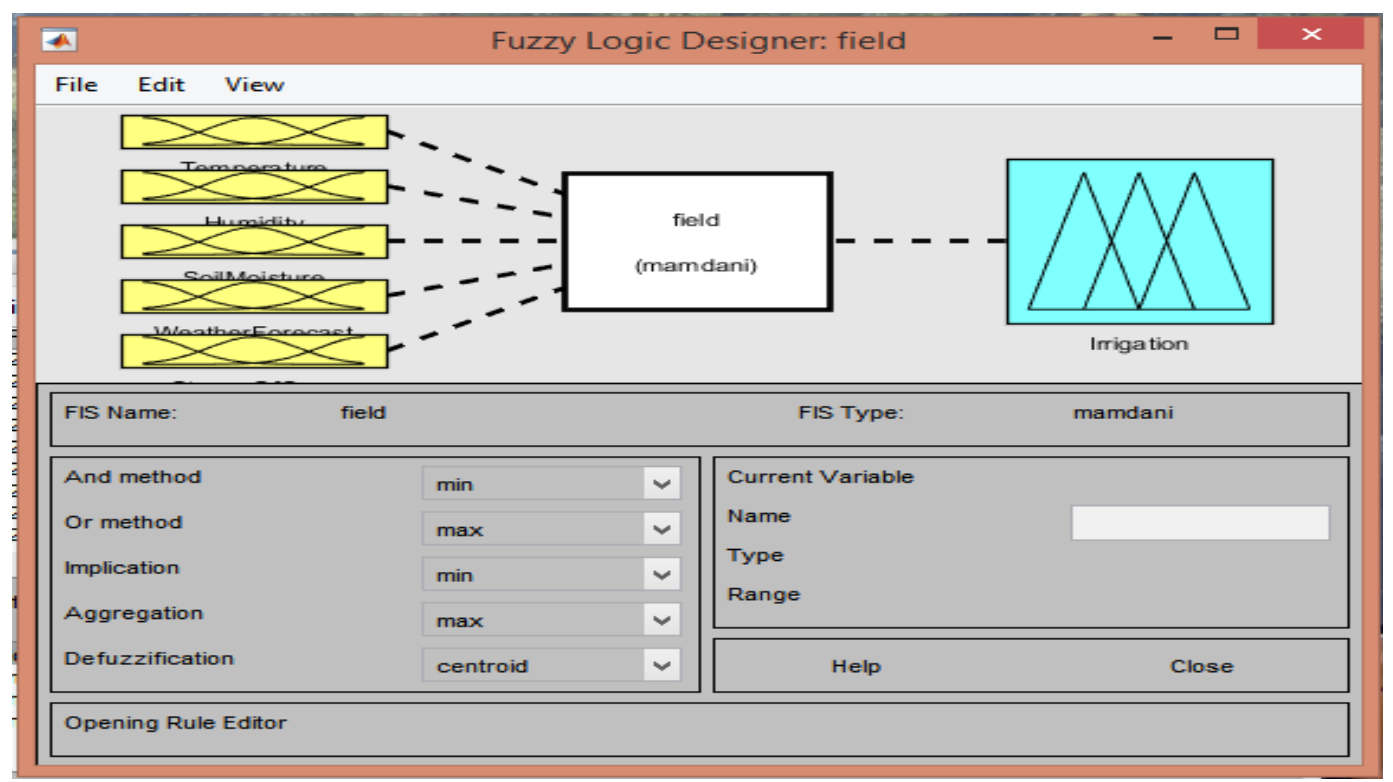

Fig. 5: Fuzzy Logic Field

In fig.6, the membership function for various input and output parameters is shown. Irrigation time in figure shows 5 different types of membership function. The type of the membership function used is trimf. A range for each membership function is specified as input. This shows the difference between various membership function.

The input parameter Temperature has three membership functions low, medium and high, which represents various temperature of the agricultural field. Similarly, Humidity has three membership functions. Soil Moisture has three membership functions namely Depth1, Depth2 and Depth3 which indicates different depth levels of agricultural field. Weather Forecast has four membership functions namely cloudy, scattered cloudy, sunny and mostly sunny which shows upcoming weather information. Stages of crop have 4 membership functions Preparation, Transplantation, Maintenance and Harvesting.

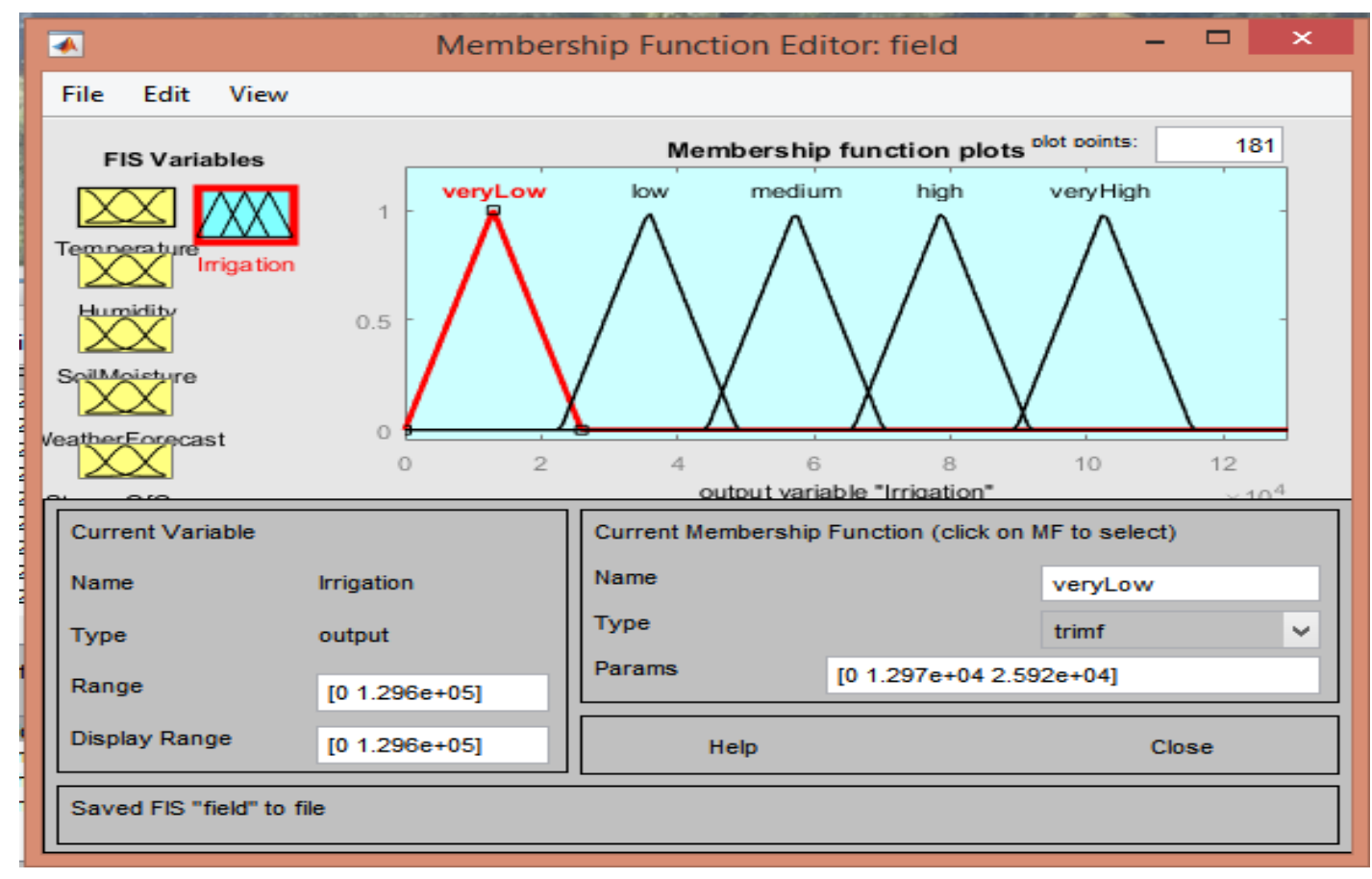

Fig. 6: Membership Function Editor

Fig. 7 shows rule editor which allows generating rules based on various combinations of input and output parameters. Fig. 8 shows the rule viewer of fuzzy toolbox, which shows the entire process from starting to end. We can move the cursor around the input lines and can view the change in the new output. 


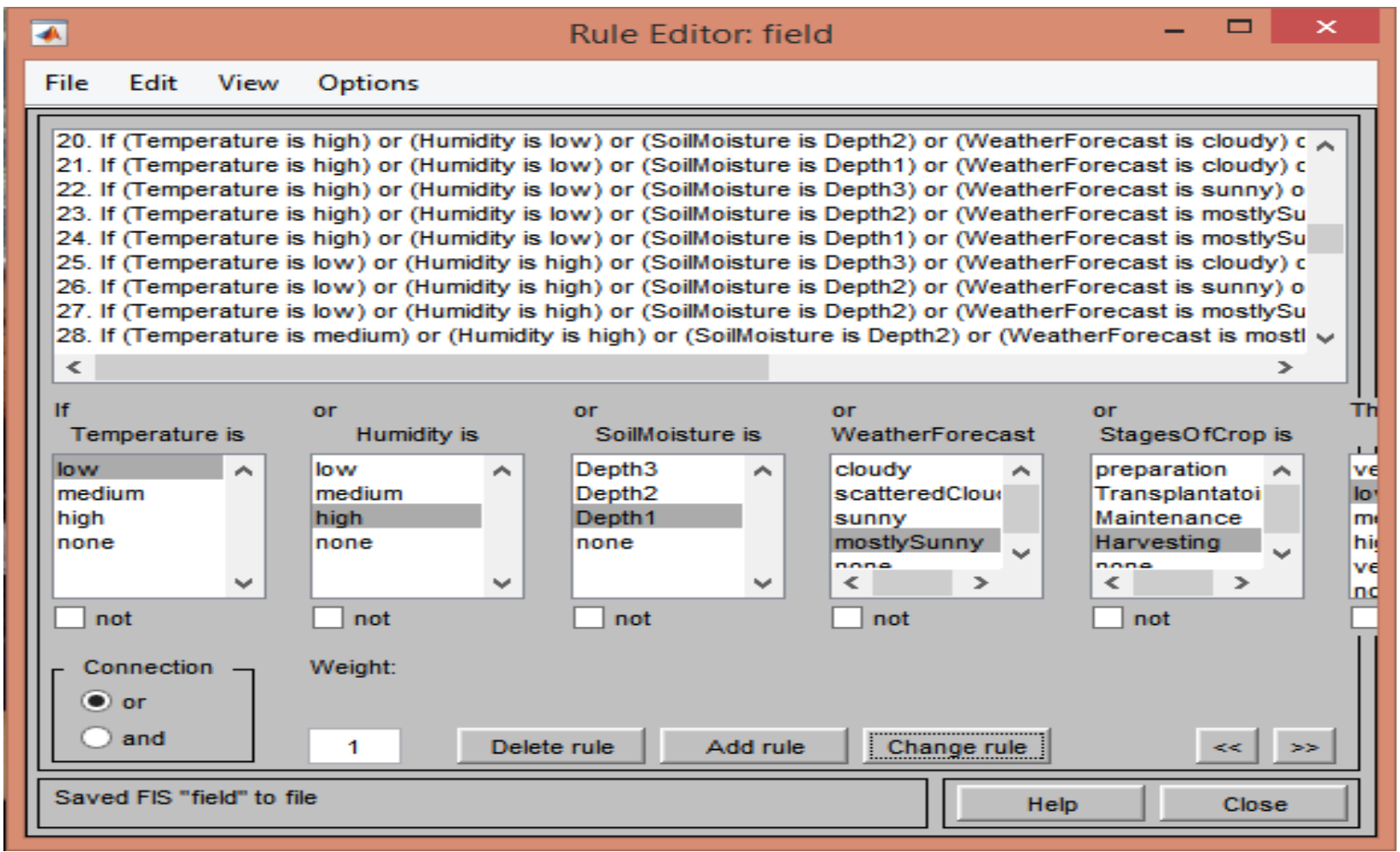

Fig. 7: Rule Editor

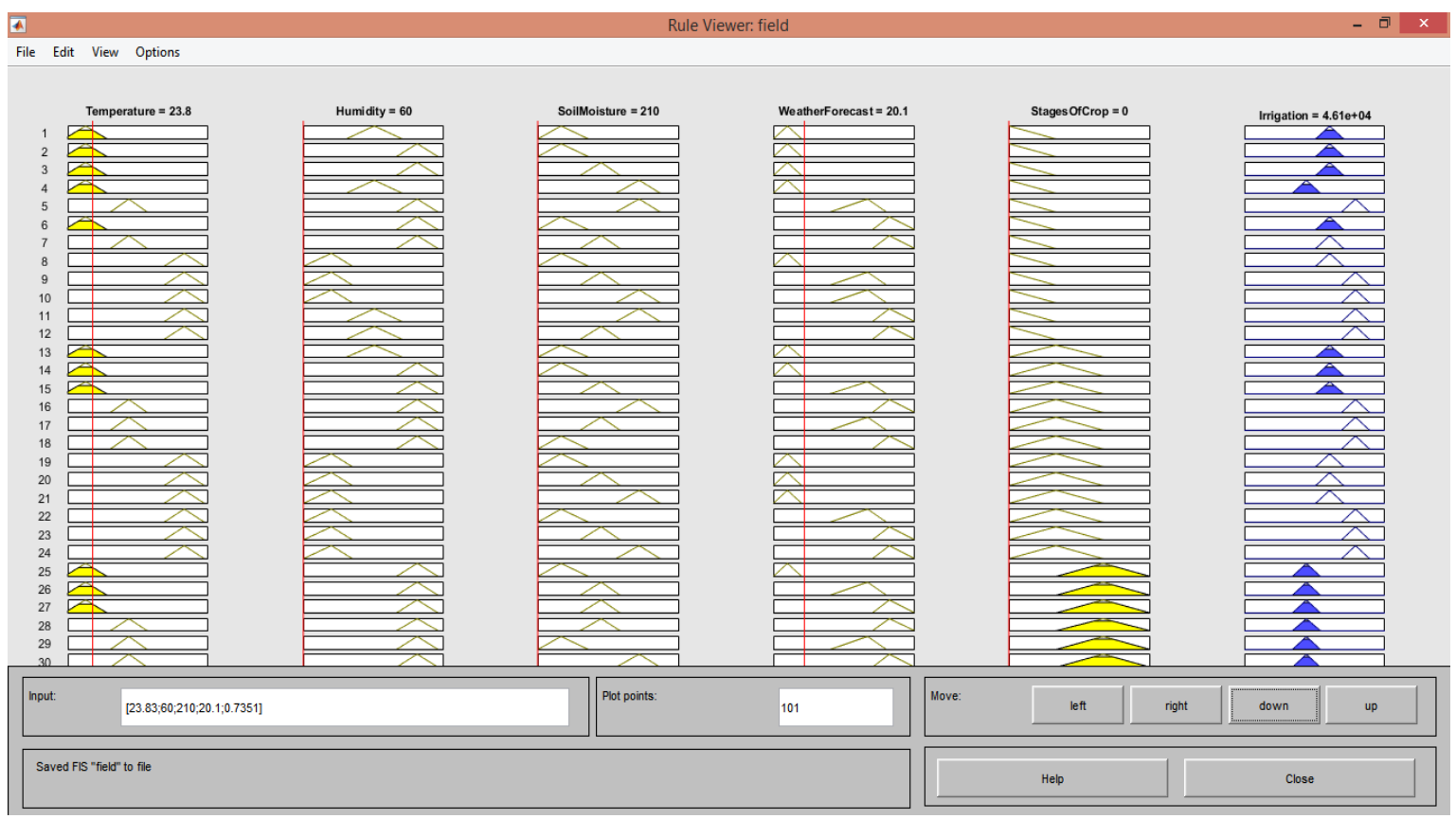

Fig. 8: Rule Viewer

With help of this fuzzy logic output, we are also going to implement artificial neural networks and verify the result with the experts to decide on the accuracy of the system.

\section{Conclusion}

A decision support system is developed to find time needed to irrigate the crop based on the information collected by the temperature, humidity and soil moisture sensors. Fuzzy logic algorithm is implemented and rules are defined. The results of fuzzy logic are to be given to adaptive neuro fuzzy algorithm. As the future work it will be validated by the expert to decide upon the accuracy level.

\section{References}

[1] Khelifa B, Amel D, Amel B, Mohamed C, Tarek B, "Smart irrigation using internet of things", InFuture Generation Communication Technology (FGCT), Fourth International Conference on 2015.

[2] Baranwal T, Pateriya PK, "Development of IoT based smart security and monitoring devices for agriculture", InCloud System and Big Data Engineering (Confluence), 6th International Conference 2016.

[3] Arvindan AN, Keerthika D, "Experimental investigation of remote control via Android smart phone of arduino-based 
automated irrigation system using moisture sensor", InElectrical Energy Systems (ICEES), 3rd International Conference on 2016

[4] Nikolidakis SA, Kandris D, Vergados DD, Douligeris C, "Energy efficient automated control of irrigation in agriculture by using wireless sensor networks", Computers and Electronics in Agriculture, 2015.

[5] Putjaika N, Phusae S, Chen-Im A, Phunchongharn P, Akkarajitsakul $\mathrm{K}$, "A control system in an intelligent farming by using arduino technology", Fifth ICT International 2016.

[6] Giusti E, Marsili-Libelli S, "A Fuzzy Decision Support System for irrigation and water conservation in agriculture", Environmental Modelling \& Software. 2015.

[7] Ojha T, Misra S, Raghuwanshi NS, "Wireless sensor networks for agriculture: The state-of-the-art in practice and future challenges", Computers and Electronics in Agriculture. 2015

[8] Navarro-Hellín H, Martínez-del-Rincon J, Domingo-Miguel R, Soto-Valles F, Torres-Sánchez R, "A decision support system for managing irrigation in agriculture", Computers and Electronics in Agriculture, 2016.

[9] M. Rajesh, Manikanthan, "ANNOYED REALM OUTLOOK TAXONOMY USING TWIN TRANSFER LEARNING", International Journal of Pure and Applied Mathematics, ISSN NO:1314-3395, Vol-116, No. 21, Oct 2017.

[10] S.V.Manikanthan, Padmapriya.T, "RECENT TRENDS IN M2M COMMUNICATIONS IN 4G NETWORKS AND EVOLUTION TOWARDS 5G', International Journal of Pure and Applied Mathematics, Vol. 115, No. 8, pp: 623-630, 2017. 